\title{
Cryoglobulinemia (Review)
}

\author{
SHIMON TAKADA ${ }^{1}$, TARO SHIMIZU ${ }^{2}$, YOSHIRO HADANO ${ }^{3}$, KENTARO MATSUMOTO $^{4}$, \\ YUKI KATAOKA $^{5}$, YU ARIMA ${ }^{6}$, TOSHIYA INOUE $^{7}$ and SUMIRE SORANO $^{8}$ \\ ${ }^{1}$ Department of General Medicine, Osaka City General Hospital, Miyakojima-ku, Osaka, Japan; \\ ${ }^{2}$ Rollins School of Public Health, Emory University, Atlanta, GA, USA; ${ }^{3}$ Shizuoka Cancer Center, Sunto-gun, \\ Shizuoka; ${ }^{4}$ National Medical Clinic, Minato-ku, Tokyo; ${ }^{5}$ Hyogo Prefectural Amagasaki Hospital, Amagasaki-shi, \\ Hyogo; ${ }^{6}$ Mimihara General Hospital, Sakai-shi, Osaka; ${ }^{7}$ Rakuwakai Otowa Hospital, Kyoto-shi, \\ Kyoto; ${ }^{8}$ Kobe University School of Medicine, Chuoku, Kobe, Japan
}

Received January 5, 2012; Accepted March 29, 2012

DOI: $10.3892 / \mathrm{mmr} .2012 .861$

\begin{abstract}
Cryoglobulins are immunoglobulins that precipitate at low temperatures and redissolve upon rewarming. Cryoglobulinemia refers to the presence of circulating cryoglobulins in serum, and generally leads to a systemic inflammatory syndrome characterized by fatigue, arthralgia, purpura, neuropathy and glomerulonephritis. The disease mainly involves small to medium-sized blood vessels and causes vasculitis due to cryoglobulin-containing immune complexes. Cryoglobulinemia is classified into three types (I, II and III) on the basis of immunoglobulin composition. Predisposing conditions include lymphoproliferative disease, collagen disease and hepatitis $\mathrm{C}$ virus (HCV) infection. The diagnosis of cryoglobulinemic syndrome is predominantly based on the laboratory demonstration of serum cryoglobulins. Treatment is often directed towards the underlying disease state. For patients with chronic HCV infection, anti-viral therapy is indicated. Intense immunosuppressive or immunomodulatory therapy, including steroids, plasmapheresis and cytotoxic agents, is reserved for organ-threatening or recalcitrant disease. In this review, we discuss the clinical characteristics of the three types of cryoglobulinemia.
\end{abstract}

\section{Contents}

1. Introduction

2. Epidemiology

3. Etiology and pathogenesis

4. Clinical manifestations

Correspondence to: Dr Shimon Takada, Department of General Medicine, Osaka City General Hospital, 2-13-22 Miyakojima-hondori, Miyakojima-ku, Osaka 534-0021, Japan

E-mail: simon.t.1012@gmail.com

Key words: cryoglobulinemia, hepatitis $\mathrm{C}$ virus, purpuric papule, vasculitis, immunoglobulins, rituximab
5. Laboratory diagnosis

6. Pathological diagnosis

7. Treatment

8. Prognosis

9. Conclusion

\section{Introduction}

Cryoglobulins are immunoglobulins (Ig) that precipitate at low temperatures and disappear when incubated at $37^{\circ} \mathrm{C}$. This phenomenon was first recognized by Wintrobe and Buell in 1933 (1). The biochemical characteristics that promote cryoprecipitation are not yet well understood. Cryoglobulins are classified into three types on the basis of their Ig composition (2). Type I is characterized by a monoclonal antibody, type II by polyclonal $\operatorname{IgG}$ and monoclonal IgM and type III by polyclonal IgG and IgM. Types II and III cryoglobulinemia are regarded as mixed as they contain two Igs of different isotypes (2). Cryoglobulinemia leads to a systemic inflammatory syndrome characterized by fatigue, arthralgia, purpura, neuropathy, and glomerulonephritis. The disease mainly involves small to medium-sized vessel vasculitis caused by cryoglobulin-containing immune complexes $(3,4)$. Cryoglobulinemia is considered to be a rare disorder, but its occurrence is strongly linked to the prevalence of hepatitis $\mathrm{C}$ virus (HCV) infection in the general population. In this review, we discuss the clinical characteristics of the three types of cryoglobulinemia.

\section{Epidemiology}

Currently, there are insufficient epidemiological studies on the prevalence of cryoglobulinemia. The incidence is reportedly $1: 100,000$ persons with a female to male ratio of 3:1 (5-9). The disease is more common in Southern Europe than in Northern Europe or North America (2,10-19). This distribution is associated with the prevalence of HCV infection. Cryoglobulins have been reported in a significant proportion of patients with chronic infections; $15-20 \%$ in patients with human immunodeficiency virus (HIV) infection and 15-25\% 
in patients with connective tissue diseases. Upon examination, $>50 \%$ of $\mathrm{HCV}$-infected individuals are found to have circulating cryoglobulins, with approximately $5 \%$ presenting with cryoglobulinemic syndrome (5-9).

\section{Etiology and pathogenesis}

Although the etiology of cryoglobulinemia is not yet completely understood, three mechanisms are considered to be major pathogenic factors: i) chronic immune stimulation and/or lymph proliferation caused by the increased production of cryoglobulins, ii) immune complex formation among cryoglobulins and/ or their antigens, and iii) insufficient clearance of cryoglobulins or their immune complexes.

Type I cryoglobulinemia. This type of cryoglobulinemia is characterized by the presence of monoclonal cryoglobulins; the production of which is due to underlying lymphoproliferative disease. Exposure to cold air induces the precipitation of Igs. These precipitates lead to inflammatory vasculitis and vessel obstruction.

Types II and III cryoglobulinemia. Hyperactivation and/or hyperproliferation of B-cells induces the selective expansion of B-cell clone(s), which in turn produce cryoglobulins $(9,20)$.

Chronic inflammatory states, including collagen diseases and viral infections (including $\mathrm{HCV}$ infection), may initiate B-cell hyperactivation. HCV directly mediates B-cell hyperactivation and causes infection via the cell surface protein CD81 (21). However, cryoglobulinemia is not always accompanied with HCV infection (22).

\section{Clinical manifestations}

Skin manifestations. Cutaneous manifestations are generally observed in type I cryoglobulinemia. Erythematous or purpuric papules (Fig. 1) of the lower extremities, infarction, hemorrhagic crusts, and ulcers are the commonly observed skin manifestations. Type I cryoglobulinemia is also more commonly associated with Raynaud's phenomenon, livedo reticularis and acrocyanosis than types II and III.

Musculoskeletal manifestations. Manifestations, such as arthralgia and myalgia are common in patients with mixed cryoglobulinemia (a combination of types II and III) $(23,24)$. In patients with type III cryoglobulinemia, the metacarpophalanges, proximal phalanges, knees and ankles are frequently affected. These lesions are often exacerbated by exposure to the cold. Musculoskeletal problems rarely accompany type I disease.

Neurological manifestations. Peripheral neuropathy, presumably caused by vasculitis, is rarely associated with clinically significant presentation (25). However, electromyographic and nerve conduction studies have revealed the presence of peripheral neuropathy in $70-80 \%$ of patients with mixed cryoglobulinemia (26-28)

Pulmonary manifestations. Respiratory manifestations, including dyspnea, cough or pleurisy, are more commonly

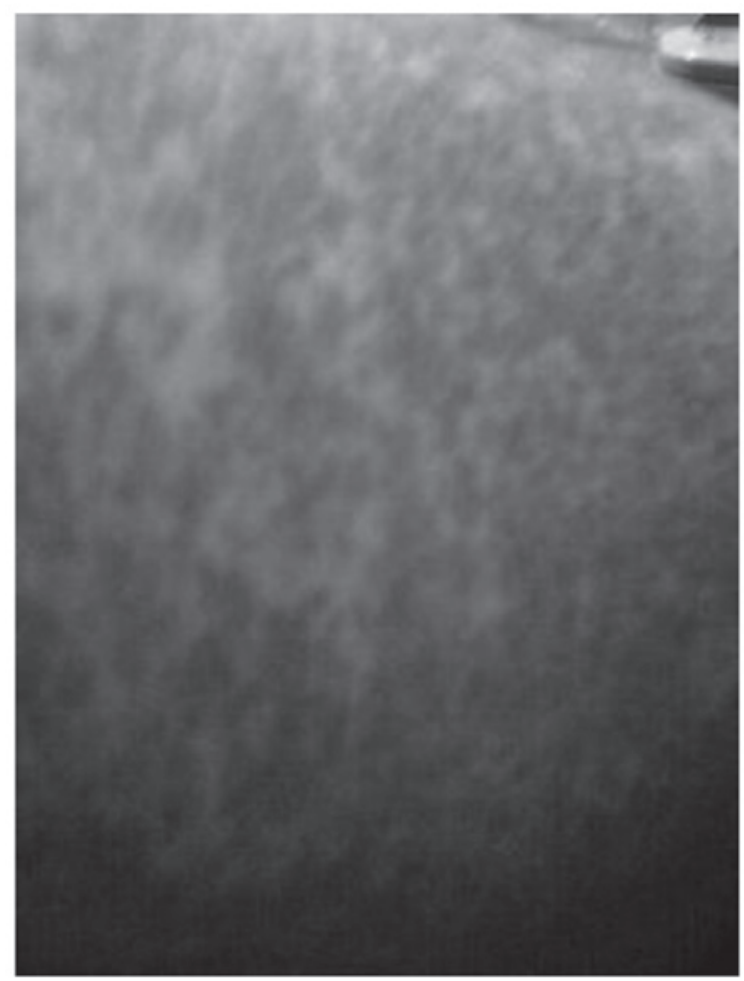

Figure 1. Skin manifestation of patient with type I cryoglobulinemia. Purpuric papules are observed on the patient's left leg.

observed in patients with mixed cryoglobulinemia than in those with type I cryoglobulinemia. These patients may have small airway disease and impairment of gas exchange.

Renal manifestations. Membranoproliferative glomerulonephritis is considered to be more common in patients with mixed cryoglobulinemia (29). Isolated proteinuria or hematuria is more commonly observed than nephritis, nephritic syndromes or acute kidney failure.

\section{Laboratory diagnosis}

The presence of a measurable level of cryoglobulins (cryocrit) remains the most reliable laboratory standard for cryoglobulin syndromes (30).

Approximately $40 \%$ of normal individuals possess detectable cryoglobulins, typically at concentrations of $<80 \mu \mathrm{g} /$ dl $(19,31)$, but these small quantities are generally insufficient to generate a detectable cryocrit. As a result, a vast majority of healthy individuals show negative results following an examination for cryoglobulins. By contrast, mixed cryoglobulins in patients with types II and III cryoglobulinemia generally produce higher concentrations (1-5 mg/dl; 0.01-0.05 g/l). Many type I cryoglobulins are present at concentrations of $>5-10 \mathrm{mg} /$ dl (0.05-0.10 g/l), with a cryocrit $>70 \%$.

Particularly in patients with a high cryoglobulin titer, cryoprecipitates during blood collection often produce cloud-like structures on peripheral blood smears, which may be mistaken for leukocytes or platelets by automated cell differential analyzers (32), or pseudoleukocytosis or pseudothrombocytosis by automated cell counters $(21,33)$. 
Diminished serum complement components may reflect an ongoing consumption by cryoglobulin-containing or -propagated immune complexes $(34,35)$. Type I cryoglobulin typically produces few serological complement abnormalities, but mixed cryoglobulins often cause reduced serum levels of total hemolytic complement (CH50) and early complement proteins $\mathrm{Clq}, \mathrm{C} 2$ and $\mathrm{C} 4$, particularly in patients with type II and III cryoglobulinemia associated with collagen vascular disease. C3 levels are generally unaffected or mildly diminished. Although modest elevations have been reported, (36) late complement components are also usually insignificantly affected.

Other tests appear to reflect a chronic inflammatory response. Acute phase reactants, including the erythrocyte sedimentation rate (ESR) and C-reactive protein concentration, are generally elevated. ESR can be markedly elevated in patients with type I cryoglobulinemia, reflecting the underlying monoclonal Ig. Mild-to-moderate hypergammaglobulinemia of IgM, IgA and/or IgG also personifies mixed cryoglobulinemia, with extreme and often monoclonal levels more indicative of type I disease. Hypogammaglobulinemia, particularly of $\operatorname{IgG}$, is rarely observed.

Autoantibodies, including antinuclear antibodies and rheumatoid factor, are often present in sera with mixed cryoglobulins $(34,37)$. In addition, blood viscosity is mildly increased (38), and a mild normochromic, normocytic anemia is often detectable (39). However, the diagnosis of cryoglobulin syndromes rests principally on the laboratory demonstration of serum cryoglobulins.

\section{Pathological diagnosis}

Type I cryoglobulinemia affects the skin, kidneys and bone marrow (12). Skin biopsies may demonstrate non-inflammatory thrombotic skin lesions with evidence of cutaneous infarction or hemorrhaging $(2,12,40)$. Sural nerve biopsies may reveal axonal degeneration caused by underlying diseases of the vasa vasorum and systemic neuronal damage (41-46). Pauci-inflammatory occlusive lesions may imply neuronal ischemia. Renal biopsies may demonstrate non-inflammatory glomerulopathies, including thrombotic and hypocellular lesions. Bone marrow examination may reveal evidence of an underlying hematological condition.

The typical pathological finding of mixed cryoglobulinemia is, which involves medium and, more often, small blood vessels $(10,16-18,47)$, and is diagnosed by a skin biopsy of recent vasculitic lesions. Mixed cryoglobulinemia is also characterized by conditions, including chronic hepatitis, membranoproliferative glomerulonephritis, peripheral neuropathy, skin ulcerations, diffuse vasculitis, and, less frequently, lymphatic and hepatic malignancies $(2,11,14,40,47-49)$.

\section{Treatment}

In clinical practice, it is difficult to treat cryoglobulin syndrome due to the wide variation in underlying disease states. Asymptomatic patients do not usually require any treatment; non-steroidal anti-inflammatory drugs (NSAIDs) are often prescribed to control mild symptoms, including general fatigue, arthralgia and myalgia.
On the contrary, patients with moderate to severe symptoms, including progressive renal failure, necrosis of the distal extremities, or uncontrolled neuropathy, need aggressive therapies, such as plasmapheresis and/or steroid and/ or cytotoxic drugs (cyclophosphamide). Plasmapheresis is conventionally required three times per week for three weeks. However, a recent study suggested that the detailed protocol of procedures and quantitative outcomes of apheresis have not yet been clarified (50).

In patients with type I cryoglobulinemia, it is imperative to treat underlying hematological malignancies or lymphoproliferative disorders. Chemotherapy and radiation therapy are indicated in such cases. A combination of oral prednisone $(1-2 \mathrm{mg} / \mathrm{kg} /$ day) and intravenous cyclophosphamide $\left(0.5-1.0 \mathrm{~g} / \mathrm{m}^{2}\right)(51)$ was reportedly used to treat a case of non-malignancy-related type I disease (52).

In order to reduce the cryoglobulin burden in patients presenting with mixed cryoglobulinemia, rapid plasma exchange should be performed in conjunction with the intravenous administration of methylprednisone daily for three days, especially in severe cases. Oral prednisone or cyclophosphamide should be added to this regimen to prevent further antibody formation (52-55).

In the majority of cases, patients are likely to present with chronic HCV infection; therefore, antiviral therapy is necessary. A suitable treatment regimen usually depends on renal function. A combination of interferon $\alpha$, pegylated interferon and ribavirin is recommended (56-59). Therapy recommended by the European League Against Rheumatism for patients presenting with normal renal function consists of a combination of pegylated interferon and ribavirin; however, for patients with renal insufficiency, monotherapy with interferon $\alpha$ is indicated since ribavirin is generally contraindicated in renal insuffiency (60).

Furthermore, rituximab therapy is recommended in patients with uncontrolled mixed cryoglobulinemia despite anti-HCV therapy. Rituximab exerts selective B-cell control and may be an alternative to immunotherapy; moreover, it is well-tolerated. In a number of studies, rituximab administered at a dose of $375 \mathrm{mg} / \mathrm{m}^{2}$ once weekly for four weeks was well-tolerated and effective in interferon $\alpha$-resistant patients with $\mathrm{HCV}$ infection (61), uncontrolled skin manifestations, peripheral neuropathy (62) and cryoglobulinemic glomerulonephritis (63). A recent study on HCV-related cryoglobulinemia suggests that a combination of rituximab, pegylated interferon $\alpha$ and ribavirin is more effective than a combination of pegylated interferon and ribavirin (64).

Finally, in patients with cryoglobulinemia associated with HBV infection, certain studies have suggested that lamivudine or entecavir may be useful (65-68).

\section{Prognosis}

The prognosis of cryoglobulinemia depends on the underlying condition(s) as well as the choice of treatment. In a literature review on the mortality of patients with mixed cryoglobulinemia, the ten-year survival rate was $<60 \%$ (69). Predictors of mortality reportedly include age, male gender, circulating cryoglobulins, immunosuppressive treatment, cutaneous ulceration, renal involvement, chronic hepatitis, widespread 
vasculitis, lymphoma and myeloproliferative diseases (70-76). The use of immunosuppressive treatment may depend on disease severity (75).

The main causes of mortality are infection, end-stage liver disease, end-stage renal disease, cardiovascular disease, neuropathy and solid tumors (including hepatic cell carcinoma) (71,73-76).

In cryoglobulinemia associated with $\mathrm{HCV}$ infection, early virologic response to interferon treatment is associated with reduced mortality (75). However, not all patients respond to interferon treatment; in certain patients, cryoglobulinemia may persist or recur after clearance of HCV RNA with antiviral therapy (77).

\section{Conclusion}

Cryoglobulinemia is considered to be a rare disorder, and epidemiological studies on its prevalence are inadequate. Clinical manifestations of cryoglobulinemia and its management vary substantially due to the wide variation in underlying diseases. Type I is characterized by monoclonal cryoglobulins produced as a result of underlying lymphoproliferative disease, type II by polyclonal IgG and monoclonal $\operatorname{IgM}$, and type III by polyclonal IgG and IgM. Types II and III are also called mixed cryoglobulinemia as they contain different isotypes, with B-cell hyperactivation and/or hyperproliferation supposedly initiated by chronic inflammatory states, including collagen disease or viral infections (including HCV). Cryoglobulinemia leads to systemic inflammatory syndrome characterized by fatigue, arthralgia, purpura, neuropathy and glomerulonephritis. The disease mainly affects small to medium-sized blood vessels and causes vasculitis due to cryoglobulin-containing immune complexes. The diagnosis of cryoglobulinemic syndrome is predominantly based on the laboratory demonstration of serum cryoglobulins, with or without associated characteristic clinical signs and symptoms. Diminished serum complement components may reflect ongoing consumption by cryoglobulin-containing or -propagated immune complexes. In clinical practice, it is difficult to treat cryoglobulin syndrome due to the wide variation in underlying conditions. Treatment is often determined on the basis of the underlying disease(s). For patients with chronic $\mathrm{HCV}$ infection, anti-viral therapy with interferon $\alpha$ and ribavirin is recommended. Patients whose symptoms are resistant to anti-HCV therapy may be suitable candidates for rituximab therapy. Intense immunosuppressive or immunomodulatory therapy, including steroids, plasmapheresis or cytotoxic agents (cyclophosphamide), is reserved for organ-threatening or recalcitrant disease. However, there are no well-delineated, evidence-based guidelines for treating cryoglobulinemia. Further research on this aspect is warranted.

\section{References}

1. Wintrobe $\mathbf{M}$ and Buell $\mathrm{M}$ : Hyperproteinemia associated with multiple myeloma: With report of a case in which an extraordinary hyperproteinemia was associated with thrombosis of the retinal veins and symptoms suggesting Raynaud's disease. Bull Johns Hopkins Hosp 52: 156-165, 1933.

2. Brouet JC, Clauvel JP, Danon F, Klein M and Seligmann M: Biologic and clinical significance of cryoglobulins. A report of 86 cases. Am J Med 57: 775-788, 1974.
3. Jennette JC, Falk RJ, Andrassy K, et al: Nomenclature of systemic vasculitides: Proposal of an international consensus conference. Arthritis Rheum 37:187-192, 1994.

4. Trendelenburg $M$ and Schifferli JA: Cryoglobulins are not essential. Ann Rheum Dis 57: 3-5, 1998.

5. Bonnet F, Pineau JJ, Taupin JL, et al: Prevalence of cryoglobulinemia and serological markers of autoimmunity in human immunodeficiency virus infected individuals: a cross-sectional study of 97 patients. J Rheumatol 30: 2005-2010, 2003.

6. García-Carrasco M, Ramos-Casals M, Cervera R, et al: Cryoglobulinemia in systemic lupus erythematosus: prevalence and clinical characteristics in a series of 122 patients. Semin Arthritis Rheum 30: 366-373, 2001.

7. Ramos-Casals M, Cervera R, Yagüe J, et al: Cryoglobulinemia in primary Sjögren's syndrome: prevalence and clinical characteristics in a series of 115 patients. Semin Arthritis Rheum 28: 200-205, 1998.

8. Cicardi M, Cesana B, Del Ninno E, et al: Prevalence and risk factors for the presence of serum cryoglobulins in patients with chronic hepatitis C. J Viral Hepat 7: 138-143, 2000.

9. Ramos-Casals M, Muñoz S, Medina F, et al: Systemic autoimmune diseases in patients with hepatitis $C$ virus infection: characterization of 1020 cases (The HISPAMEC Registry). J Rheumatol 36: 1442-1448, 2009.

10. Ferri C, Zignego AL and Pileri SA: Cryoglobulins (review). J Clin Pathol 55: 4-13, 2002.

11. Meltzer M, Franklin EC, Elias K, McCluskey RT and Cooper N: Cryoglobulinemia - A clinical and laboratory study. II. Cryoglobulins with rheumatoid factor activity. Am J Med 40: 837-856, 1966.

12. Gorevic PD, Kassab HJ, Levo Y, Kohn R, Meltzer M, Prose P and Franklin E: Mixed cryoglobulinemia: clinical aspects and long-term follow-up of 40 patients. Am J Med 69: 287-308, 1980.

13. Invernizzi F, Galli M, Serino G, Monti G, Meroni PL, Granatieri C and Zanussi C: Secondary and essential cryoglobulinemias. Frequency, nosological classification, and long-term follow-up. Acta Haematol 70: 73-82, 1983.

14. Monti G, Galli M, Invernizzi F, Pioltelli P, Saccardo F, Monteverde A, Pietrogrande M, Renoldi P, Bombardieri S, Bordin $\mathrm{G}$, et al: Cryoglobulinaemias: a multi-center study of the early clinical and laboratory manifestations of primary and secondary disease. GISC. Italian Group for the Study of Cryoglobulinaemias. QJM 88: 115-126, 1995.

15. Dammacco F and Sansonno D: Mixed cryoglobulinemia as a model of systemic vasculitis. Clin Rev Allergy Immunol 15: 97-119, 1997.

16. Lamprecht P, Gause A and Gross WL: Cryoglobulinemic vasculitis. Arthritis Rheum 42: 2507-2516, 1999.

17. Ferri C, Antonelli A, Mascia MT, Sebastiani M, Fallahi P, Ferrari D, Pileri SA and Zignego AL: HCV-related autoimmune and neoplastic disorders: the HCV syndrome. Dig Liver Dis 39: S13-S21, 2007.

18. Ferri C, Antonelli A, Mascia MT, Sebastiani S, Fallhai P, Ferrari D, Giunti M, Pileri SA and Zignego AL: B-cells and mixed cryoglobulinemia. Autoimmun Rev 7: 114-120, 2007.

19. Mascia MT, Ferrari D, Campioli D, Sandri G, Mussini C and Ferri C: Non HCV-related mixed cryoglobulinemia. Dig Liver Dis 39: S61-S64, 2007.

20. Ferraccioli GF, De Vita S, Casatta L, et al: Autoimmune connective tissue disease, chronic polyarthritides and B cell expansion: risks and perspectives with immunosuppressive drugs. Clin Exp Rheumatol 14: S71-S80, 1996.

21. Pileri P, Uematsu Y, Campagnoli S, et al: Binding of hepatitis C virus to CD81. Science 282: 938-941, 1998.

22. Schott P, Polzien F, Müller-Issberner A, et al: In vitro reactivity of cryoglobulin IgM and $\operatorname{IgG}$ in hepatitis $\mathrm{C}$ virus-associated mixed cryoglobulinemia. J Hepatol 28: 17-26, 1998.

23. Ramos-Casals M, Trejo O, García-Carrasco M, et al: Mixed cryoglobulinemia: new concepts. Lupus 9: 83-91, 2000.

24. Weinberger A, Berliner S and Pinkhas J: Articular manifestations of essential cryoglobulinemia. Semin Arthritis Rheum 10: 224-229, 1981.

25. Santoro L, Manganelli F, Briani C, Giannini F, Benedetti L, Vitelli E, Mazzeo A, Beghi E; HCV Peripheral Nerve Study Group: Prevalence and characteristics of peripheral neuropathy in hepatitis C virus population. J Neurol Neurosurg Psychiatry 77: 626-629, 2006

26. Montagnino G: Reappraisal of the clinical expression of mixed cryoglobulinemia: Springer Semin Immunopathol 10: 1-19, 1988 . 
27. Ferri C, La Civita L, Cirafisi C, et al: Peripheral neuropathy in mixed cryoglobulinemia. clinical and electrophysiologic investigations. J Rheumatol 19: 889-895, 1992.

28. Gemignani F, Pavesi G, Fiocchi A, et al: Peripheral neuropathy in essential mixed cryoglobulinaemia. J Neurol Neurosurg Psychiatry 55: 116-120, 1992.

29. Roccatello D, Fornasieri A, Giachino O, Rossi D, Beltrame A, Banfi G, Confalonieri R, Tarantino A, Pasquali S, Amoroso A, Savoldi S, Colombo V, Manno C, Ponzetto A, Moriconi L, Pani A, Rustichelli R, Di Belgiojoso GB, Comotti C and Quarenghi MI: Multicenter Study on Hepatitis C Virus-Related Cryoglobulinemic Glomerulonephritis. Am J Kidney Dis 49: 69-82, 2006

30. Antonelli A, Ferri C, Fallahi P, Sebastiani M, Nesti C, Barani L, Barale R and Ferrannini E: Type 2 diabetes in hepatitis C-related mixed cryoglobulinaemia patients. Rheumatology (Oxford) 43: 238-240, 2004

31. Pozzato G, Mazzaro C, Crovatto M, Modolo ML, Ceselli S, Mazzi G, Sulfaro S, Franzin F, Tulissi P, Moretti M and Santini GF: Low-grade malignant lymphoma, hepatitis $C$ virus infection, and mixed cryoglobulinemia. Blood 84: 3047-3053, 1994.

32. Zignego AL, Macchia D, Monti M, Thiers V, Mazzetti M, Foschi M, Maggi E, Romagnani S, Gentilini P and Bréchot C: Infection of peripheral mononuclear blood cells by hepatitis $\mathrm{C}$ virus. J Hepatol 15: 382-386, 1992.

33. Zignego AL, Ferri C, Pileri SA, Caini P, Bianchi FB; for the Italian Association of the Study of Liver Commission on Extrahepatic Manifestations of HCV infection: Extrahepatic manifestations of Hepatitis C Virus infection. a general overview and guidelines for a clinical approach. Dig Liver Dis 39 :2-17, 2007.

34. Monteverde A, Ballarè M and Pileri S: Hepatic lymphoid aggregates in chronic hepatitis $\mathrm{C}$ and mixed cryoglobulinemia. Springer Semin Imunopathol 19: 99-110, 1997.

35. Sansonno D, Tucci FA, De Re V, Lauletta G, Montrone M, Libra M and Dammacco F: HCV-associated B cell clonalities in the liver do not carry the $t(14 ; 18)$ chromosomal translocation. Hepatology 42: 1019-1027, 2005.

36. Giannini C, Giannelli F and Zignego AL: Association between mixed cryoglobulinemia, translocation $(14 ; 18)$, and persistence of occult HCV lymphoid infection after treatment. Hepatology 42: 1019-1027, 2005.

37. Manganelli P, Salaffi F, Subiaco S, Carotti M, Cervini C, Consigli G, Majori $\mathrm{M}$ and Pesci A: Bronchoalveolar lavage in mixed cryoglobulinaemia associated with hepatitis $\mathrm{C}$ virus. $\mathrm{Br} \mathrm{J}$ Rheumatol 35: 978-982, 1996.

38. Machida K, Cheng KT, Sung VM, Shimodaira S, Lindsay KL, Levine AM, Lai MY and Lai MM: Hepatitis C virus induces a mutator phenotype: enhanced mutations of immunoglobulin and protooncogenes. Proc Natl Acad Sci USA 101: 4262-4267, 2004

39. Migliaresi S, Bresciani A, Ambrosone L, Spera M, Barbarulo D, Lombari V, Pirozzi G, Borgia G, Lombardi ML, Tirri G and Manzo C: Increased serum concentrations of soluble HLA-class I antigens in hepatitis $\mathrm{C}$ virus related mixed cryoglobulinaemia. Ann Rheum Dis 59: 20-25, 2000.

40. Cohen, P, Nguyen, QT, Deny P, et al: Treatment of mixed cryoglobulinemia with recombinant interferon alpha and adjuvant therapies: A prospective study on 20 patients. Ann Med Interne (Paris) 147: 81-86, 1996.

41. Garcia-Bragado, F, Fernandez, JM, Navarro, C, et al: Periphera neuropathy in essential mixed cryoglobulinemia. Arch Neurol 45 1210-1214, 1988

42. Chad, D, Pariser, K, Bradley, WG, et al: The pathogenesis of cryoglobulinemic neuropathy. Neurology 32: 725-729, 1982.

43. Nemni, R, Corbo, M, Fazio, R, et al: Cryoglobulinaemic neuropathy: A clinical, morphological and immunocytochemical study of 8 cases. Brain 111: 541-552, 1988.

44. Tredici, G, Petruccioli, MG, Cavaletti, G, et al: Sural nerve bioptic findings in essential cryoglobulinemic patients with and without peripheral neuropathy. Clin Neuropathol 11: 121-127, 1992.

45. Bonetti, B, Invernizzi, F, Rizzuto, N, et al: T-cell-mediated epineurial vasculitis and humoral-mediated microangiopathy in cryoglobulinemic neuropathy. J Neuroimmunol 73: 145-154, 1997.

46. Cavaletti, G, Petruccioli, MG, Crespi V, et al: A clinicopathological and follow up study of 10 cases of essential type II cryoglobulinaemic neuropathy. J Neurol Neurosurg Psychiatry 53: 886-889, 1990.

47. Ferri $C$ and Mascia MT: Cryoglobulinemic vasculitis: Review. Curr Opin Rheumatol 18: 54-63, 2006.
48. Abel G, Zhang QX and Agnello V: Hepatitis $\mathrm{C}$ virus infection in type II mixed cryoglobulinemia. Arthritis Rheum 36:1341-1349, 1993.

49. Ferri C, La Civita L, Longombardo G, et al: Mixed cryoglobulinemia: a cross-road between autoimmune and lymphoproliferative disorders. Lupus 7: 275-279, 1998.

50. Rockx MA and Clark WF: Plasma exchange for treating cryoglobulinemia: a descriptive analysis. Transfus Apher Sci 42: 247-251, 2010

51. Ristow SC, Griner PF, Abraham GN and Shoulson I: Reversal of systemic manifestations of cryoglobulinemia: Treatment with melphalan and prednisone. Arch Intern Med 136: 467-470, 1976.

52. Frankel AH, Singer DR, Winearls CG, et al: Type II essential mixed cryoglobulinaemia: presentation, treatment and outcome in 13 patients. Q J Med 82: 101-124, 1992.

53. D'Amico G, Colasanti G, Ferrario F and Sinico RA: Renal involvement in essential mixed cryoglobulinemia. Kidney Int 35: 1004-1014, 1989

54. Madore F, Lazarus JM and Brady HR: Therapeutic plasma exchange in renal diseases. J Am Soc Nephrol 7: 367-386, 1996.

55. Guillevin L and Pagnoux C: Indications of plasma exchanges for systemic vasculitides. Ther Apher Dial 7: 155-160, 2003.

56. Misiani R, Bellavita P, Baio P, et al: Successful treatment of HCV-associated cryoglobulinaemic glomerulonephritis with a combination of interferon-alpha and ribavirin. Nephrol Dial Transplant 14: 1558-1560, 1999.

57. Zuckerman E, Keren D, Slobodin G, et al: Treatment of refractory, symptomatic, hepatitis $\mathrm{C}$ virus related mixed cryoglobulinemia with ribavirin and interferon-alpha. J Rheumatol 27: 2172-2178, 2000.

58. Mazzaro C, Zorat F, Caizzi M, et al: Treatment with peginterferon alfa- $2 b$ and ribavirin of hepatitis $C$ virus-associated mixed cryoglobulinemia: a pilot study. J Hepatol 42: 632-638, 2005.

59. Cacoub P, Saadoun D, Limal N, et al: PEGylated interferon alfa-2b and ribavirin treatment in patients with hepatitis $C$ virus-related systemic vasculitis. Arthritis Rheum 52: 911-915, 2005.

60. Mukhtyar C, Guillevin L, Cid MC, Dasgupta B, de Groot K, Gross W, Hauser T, Hellmich B, Jayne D, Kallenberg CG, Merkel PA, Raspe H, Salvarani C, Scott DG, Stegeman C, Watts R, Westman K, Witter J, Yazici H, Luqmani R; European Vasculitis Study Group: EULAR recommendations for the management of primary small and medium vessel vasculitis. Ann Rheum Dis 68: 310-317, 2009

61. Sansonno D, De Re V, Lauletta G, et al: Monoclonal antibody treatment of mixed cryoglobulinemia resistant to interferon alpha with an anti-CD20. Blood 101: 3818-3826, 2003.

62. Zaja F, De Vita S, Mazzaro C, et al: Efficacy and safety of rituximab in type II mixed cryoglobulinemia. Blood 101: 3827-3834, 2003.

63. Quartuccio L, Soardo G, Romano G, et al: Rituximab treatment for glomerulonephritis in HCV-associated mixed cryoglobulinaemia: efficacy and safety in the absence of steroids. Rheumatology (Oxford) 45: 842-846, 2006.

64. Saadoun D, Resche Rigon M, Sene D, Terrier B, Karras A, Perard L, Schoindre Y, Coppéré B, Blanc F, Musset L, Piette JC, Rosenzwajg M and Cacoub P: Rituximab plus Peg-interferon-alpha/ribavirin compared with Peg-interferon a/ribavirin in hepatitis C-related mixed cryoglobulinemia. Blood 116: 326-334, 2010

65. Sawabe T, Uenotsuchi T, Imafuku S, et al: Remission of hepatitis B virus-related vasculitis with lamivudine. Ann Intern Med 140: 672-673, 2004

66. Stecevic V, Pevzner MM and Gordon SC: Successful treatment of hepatitis B-associated vasculitis with lamivudine. J Clin Gastroenterol 36: 451, 2003.

67. Kawakami T, Ooka S, Mizoguchi M, et al: Remission of hepatitis B virus-related cryoglobulinemic vasculitis with entecavir. Ann Intern Med 149: 911-912, 2008.

68. Enomoto M, Nakanishi T, Nakamishi T, et al: Entecavir to treat hepatitis B-associated cryoglobulinemic vasculitis. Ann Intern Med 149: 912-913, 2008.

69. Della Rossa A, Marchi F, Catarsi E, et al: Mixed cryoglobulinemia and mortality: a review of the literature. Clin Exp Rheum 26: S105-S108, 2008.

70. Kordossis T, Sipsas NV, Kontos A, et al: Mixed cryoglobulinemia is associated with increased risk for death, or neoplasia in HIV-1 infection. Eur J Clin Invest 31: 1078-1082, 2001. 
71. Ferri C,Sebastiani M, Giuggioli D, et al: Mixed cryoglobulinemia: demographic, clinical, and serologic features and survival in 231 patients. Semin Arthritis Rheum Jun 33: 355-374, 2004

72. Bryce AH, Kyle RA, Dispenzieri A, et al: Natural history and therapy of 66 patients with mixed cryoglobulinemia. Am J Hematol Jul 81: 511-518, 2006.

73. Saadoun D, Resche-Rigon M, Thibault V, et al: Antiviral therapy for hepatitis C virus - associated mixed cryoglobulinemia vasculitis: a long-term followup study. Arthritis Rheum 54: 3696-3706, 2006.

74. Saccardo E, Novati P, Sironi D, et al: Causes of death in symptomatic cryoglobulinemia: 30 years of observation in a Department of Internal Medicine. Dig Liver Dis 39: S52-S54, 2007.
75. Landau DA, Scerra S, Sene D, et al: Causes and predictive factors of mortality in a cohort of patients with hepatitis $C$ virus-related cryoglobulinemic vasculitis treated with antiviral therapy. J Rheumatol Mar 37: 615-621, 2010.

76. Della Rossa A, Tavoni A, D'Ascanio A, et al: Mortality rate and outcome factors in mixed cryoglobulinaemia: the impact of hepatitis C virus. Scand J Rheumatol Mar 39: 167-170, 2010.

77. Levine JW, Gota C, Fessler BJ, et al: Persistent cryoglobulinemic vasculitis following successful treatment of hepatitis $\mathrm{C}$ virus. J Rheumatol 32: 1164-1167, 2005. 